Cahiers d'études africaines

$229 \mid 2018$

Varia

\title{
ALlOU, Kouamé René. - Les Nzema. Un peuple akan de Côte d'Ivoire et du Ghana
}

\section{Mariano Pavanello}

\section{OpenEdition}

Journals

Édition électronique

URL : https://journals.openedition.org/etudesafricaines/22006

DOI : 10.4000/etudesafricaines.22006

ISSN : 1777-5353

\section{Éditeur}

Éditions de l'EHESS

\section{Édition imprimée}

Date de publication : 15 mars 2018

Pagination : 233-236

ISBN : 978-2-7132-2741-7

ISSN : 0008-0055

\section{Référence électronique}

Mariano Pavanello, " AlLou, Kouamé René. - Les Nzema. Un peuple akan de Côte d'Ivoire et du Ghana », Cahiers d'études africaines [En ligne], 229 | 2018, mis en ligne le 15 mars 2018, consulté le 22 avril 2022. URL : http://journals.openedition.org/etudesafricaines/22006 ; DOI : https://doi.org/10.4000/ etudesafricaines.22006

Ce document a été généré automatiquement le 22 avril 2022.

(C) Cahiers d'Études africaines 


\title{
ALloU, Kouamé René. - Les Nzema. Un peuple akan de Côte d'Ivoire et du Ghana
}

\author{
Mariano Pavanello
}

\section{RÉFÉRENCE}

ALLou, Kouamé René. - Les Nzema. Un peuple akan de Côte d'Ivoire et du Ghana. Paris,

L'Harmattan, 2013, 238 p., bibl.

1 L'Harmattan est une maison d'édition qui a le mérite d'avoir permis à beaucoup d'auteurs et de spécialistes africains de publier un nombre important d'ouvrages, dont la plupart sont de qualité remarquable. Avec Les Nzema. Un people akan de Côte d'Ivoire et du Ghana par Kouamé René Allou, L'Harmattan publie en 2013 un travail dont la valeur est douteuse, tant du côté scientifique que de celui de l'édition de l'ouvrage. Ce livre s'occupe d'une société qui a fait l'objet de recherches ethnologiques et historiques de deux générations de spécialistes italiens depuis soixante ans. L'ethnographie nzema est, entre autres, le produit de l'École ethnologique de l'Université de Rome "La Sapienza » et de nombreux chercheurs travaillant sur le terrain, notamment depuis 1954, et qui ont publié plus d'une centaine d'ouvrages scientifiques dont un bon nombre de textes en français et en anglais, ainsi que d'autres publications dans des revues scientifiques internationales ${ }^{1}$. À cela, il faut ajouter que l'histoire politique et sociale du maanle nzema, du XVI ${ }^{e}$ au XVIII ${ }^{e}$ siècle, est l'œuvre d'un historien africaniste italien, Pierluigi Valsecchi, qui travaille sur les archives africaines, européennes et sur le terrain depuis les années $1980^{2}$. Kouamé René Allou (né à Abidjan en 1960), maître de conférence à l'Université Félix Houphouët-Boigny d'Abidjan, se qualifie spécialiste des sociétés akan, bien qu'il semble ignorer complètement la tradition des études italiennes; sur l'histoire et la culture nzema il signale uniquement l'article de Denise Paulme ${ }^{3}$ sur le festival Kundum à Grand Bassam, et une étude préliminaire qui date de 1986 de Pierluigi 
Valsecchi ${ }^{4}$ portant sur des document d'archives. Il présente son livre sur l'histoire et la culture des Nzema comme si rien n'avait été produit auparavant ; qui plus est, il nous propose une reconstruction de l'histoire des Nzema du Ghana et de la Côte d'Ivoire fondée essentiellement sur des sources secondaires : le mémoire de maitrise de James Y. Ackah (1965) qui traite des événements de la seconde moitié du XIX siècle, la thèse de doctorat d'État d'Henriette Diabaté (1984) sur le Sannvin, et trois ouvrages d'Albert Van Dantzig $(1978,1979,1980)$ sur la présence hollandaise en Guinée et sur le fort St. Antoine d'Axim ${ }^{5}$. Les références aux archives sont tirées de ces sources secondaires. Allou fonde, tout d'abord, son argumentation historique sur l'hypothèse qu'une chefferie appelée Guiomeré - qui apparaît dans la carte D'Anville $(1729)^{6}$, et qu'il interprète comme étant une forme d'altération introduite par les Européens du nom local Adjomolo - est la structure politique originaire des Nzema; elle aurait existé depuis le $\mathrm{XV}^{\mathrm{e}}$ siècle jusqu'à la dissolution de l'ancien royaume de Kaku Aka (1848) et la création des deux chefferies nzema de l'Est et de l'Ouest ${ }^{7}$. Pour affirmer la continuité de l'État nzema à partir du XV siècle, il ne s'appuie sur aucune base documentaire, ni sur des éléments de vérification des traditions orales, donc en dehors de tout cadre critique acceptable. Son argument - qui s'écarte des récentes analyses présentées par Valsecchi - est développé à plusieurs reprises et s'appuie sur des listes dynastiques tirées de sources non contrôlées dont la chronologie est en grande partie incertaine ou inventée. Une telle position cache peut-être une arrière-pensée politique liée aux mouvements de revendication de la royauté précoloniale des Nvavile ${ }^{8}$ contre les deux dynasties de chefs suprêmes coutumiers d'Elemgbenle et de Jomoro partageant le pouvoir, dit traditionnel, sur le pays nzema depuis la moitié du XIX ${ }^{\mathrm{e}}$ siècle. Un deuxième aspect de l'entreprise historiographique d'Allou concerne la reconstruction du processus de peuplement du territoire qui va de la bouche de l'Ankobra, à l'Est, jusqu'à l'embouchure de la grande lagune Dwen (Ehy-Tendo), à l'Ouest. L'exposé qui en résulte est un mélange de récits de migrations et de fondations de villages que Kouamé René Allou utilise dans leur sens littéral et donc de façon non critique. Pour la plupart, ce sont des récits recueillis et publiés par des intellectuels locaux ${ }^{9}$; ils sont complètement décontextualisés et sans aucun élément de vérification. L'architecture d'une telle entreprise historiographique, donc, n'est pas seulement faible, mais presque dénuée de tout fondement méthodologique.

2 Bien que Les Nzema. Un peuple akan de Côte d'Ivoire et du Ghana soit un ouvrage essentiellement historique, il ne manque pas d'éléments ethnographiques. Allou propose plusieurs reconstructions d'aspects fondamentaux de la culture et de la vie sociale des Nzema qui s'avèrent souvent le fruit d'informations partielles, ou erronées. Par exemple, à la page 36, il affirme que chez les Akan les «les abusuan sont exogames "; il a parfaitement raison en ce qui concerne les Asante et autres sociétés akan, mais pour ce qui est des Nzema, il faut préciser que les mmusua (pl. d'abusua), en tant que clans, ne sont pas exogamiques, bien au contraire, l'exogamie étant strictement observée à l'intérieur des mmusua en tant que lignages. À la page 37, l'auteur affirme: "L'abusuan a deux aspects. L'abusuan large ou clan que les Nzema appellent assalo abusuan et l'abusuan réduit ou lignage suakunlu abusuan. » Allou reproduit ainsi l'affirmation de Grottanelli $(1977$, pp. 36, 42) qui pourtant n'est jamais cité. Je ne doute pas qu'il sache que l'expression asalo abusua désigne le cercle extérieur du matrilignage, c'est-à-dire l'ensemble des matrilignées d'origine servile, adoptées ou prises en gage, et que suakunlu abusua s'applique uniquement au noyau soi-disant aristocratique (dehele) du matrilignage. Néanmoins, il répète un stéréotype qui sert à 
cacher la réalité de l'héritage de l'esclavage domestique en pays nzema. Si Allou avait consulté le Journal des africanistes paru en 2005 sous la direction de Gérard Chouin, Claude-Hélène Perrot et Gérard Pescheux ${ }^{10}$, il aurait pris conscience que le débat historiographique et ethnologique sur les Akan, et les Nzema en particulier, avait déjà remis en question plusieurs des certitudes qu'il continue à entériner dans son livre.

Pour finir, on ne peut pas passer sous silence que l'ouvrage de Kouamé René Allou est truffé d'une quantité de coquilles qui fatiguent la lecture. Par ailleurs, un certain nombre de références bibliographiques citées dans le texte ou dans les notes sont incomplètes, carrément erronées ou ne sont pas présentes dans la bibliographie de fin d'ouvrage.

\section{NOTES}

1. Vinigi L. Grottanelli (1912-1983) est l'ethnologue italien qui a réalisé l'ethnographie nzema et a dirigé la Mission ethnologique italienne au Ghana de 1954 à 1975. Entre 1958 et 1988 , il a publié plusieurs essais en anglais et en français et une vaste monographie en italien: V. L. GROTTANeLli, Una società guineana, gli Nzema: vol. 1, I fondamenti della cultura, Torino, Boringhieri, 1977 ; Una società guineana : gli Nzema : vol. 2, Ordine morale e salvezza terrena, Torino, Boringhieri, 1978. Parmi ses élèves et collaborateurs, Italo Signorini a analysé la terminologie de parenté nzema: I. SIGNORINI, « Nzema Kinship Terminology », Paideuma, vol. 24, 1978, pp. 111-119. L'auteur de ce compte rendu a dirigé la Mission ethnologique italienne au Ghana (MEIG) de 1989 à 2013 et a publié de nombreuses études sur les Nzema dont trois en français dans les Cahiers d'Études africaines et le Journal des africanistes (M. PAVANELLO, «Parenté, catégories économiques et théorie du pouvoir. Le cas des Nzema du Ghana sud occidental ", Cahiers d'Études africaines, XXXVI (3), 143, 1996, pp. 373-396 ; «L'événement et la parole. La conception de l'histoire et du temps historique dans les traditions orales africaines: le cas des Nzema », Cahiers d'Études africaines, XLIII (3), 171, 2003, pp. 461-481; "Clan, lignage et mariage en pays Nzema: une reconsidération", Journal des africanistes ("Approches croisées des mondes akan, I, Histoire, Anthropologie »), 75, 2005, pp. 209-232. Voir également l'article du dictionnaire Larousse de M. PAVANELLo, « Nzema », in J.-C. TAMISIER (dir.), Dictionnaire des peuples, Paris, Larousse, 1998.

2. P. VALSECCHI, I signori di Appolonia. Poteri e formazione dello Stato in Africa occidentale fra XVI e XVIII secolo, Rome, Carocci, 2002 ; Power and State Formation in West Africa : Appolonia from the Sixteenth to the Eighteenth Century, New York, Palgrave Macmillan, 2011.

3. D. PAULmE, «Un rituel de fin d'année chez les Nzema de Grand-Bassam », Cahiers d'Études africaines, X (2), 38, 1970, pp. 189-202.

4. P. VALSECCHI, «Lo Nzema fra egemonia asante ed espansione europea nella prima metà del XIX Secolo », Africa. Rivista trimestrale di studi e documentazione dell'Istituto ItaloAfricano (Roma), 41 (4), 1986, pp. 221-264. 
5. J. Ү. АСКАН [NYAMEKE AKA], Kaku Ackah and the Split of Nzema, Master's Thesis, Legon, University of Ghana, Institute of African Studies, 1965; H. DIABATÉ, Le Sannvin. Un royaume akan de la Côte d'Ivoire (1701-1901). Sources orales et histoire, Thèse de doctorat d'État, Paris, Université de Paris I Panthéon-Sorbonne, 1984 ; A. VAN DANTZIG, The Dutch and the Guinea Coast, 1674-1742: A Collection of Documents from the General State Archive at The Hague, Accra, Ghana Academy of Arts and Sciences, 1978 ; « La "jurisdiction" du Fort Saint Antoine d'Axim ", Revue Française d'Histoire d'Outre-Mer, 66 (242-243), 1979, pp. 223-236; Les Hollandais sur la côte de Guinée à l'époque de l'essor de l'Ashanti et du Dahomey, 1680-1740, Paris, Société française d'histoire d'outre-mer, 1980.

6. Paris, Bibliothèque nationale de France, GE C-6122 (A).

7. Eastern Nzema et Western Nzema States (aujourd'hui «Traditional Areas») correspondant aux deux districts administratifs d'Elemgbenle et Jomoro de la Western Region du Ghana.

8. Voir aussi P. VALSECCHI, «The Fall of Kaku Aka: Social and Political Change in the Mid-Nineteenth-Century Western Gold Coast », Journal of West African History, 2 (1), 2016, pp. 1-26.

9. Voir P. A. KWESI ABOAGYE, Nzema AneE ne Anwo Mgbanyidweke [Nzema Language and Stories of the Ancestors], Accra, Bureau of Ghana Languages, 1973 ; et surtout les trois volumes de J. A. essuah, Mekakye Bie, I, II, III, Cape Coast, Catholic Mission Press, 1958-1962.

10. G. CHOUIN, C.-H. PERROT \& G. PESCHEUX (DIR.), numéro spécial « Approches croisés des mondes Akan, I (I, Histoire, Anthropologie) », Journal des africanistes, 75 (1), 2005. Sur le problème d'asalo et suakunlu abusua, voir M. PAVANELLO, "Clan, lignage et mariage en pays nzema (Ghana) », Journal des africanistes, 75 (1), 2005, pp. 223-227. 